\title{
Sn Isotopes of Bjurböle (LL4) chondrules and matrix: implications for complementarity and chondrule thermal processing
}

\author{
FRANCESCA MIROLO, TRACY RUSHMER AND JOHN \\ CREECH
}

Macquarie University

Presenting Author: francesca.mirolo@hdr.mq.edu.au

Sn stable isotopes are a promising new lens through which to examine the partial heating, thermal metamorphism and collisional events that have occurred to chondrite components. $\mathrm{Sn}$ is of partial siderophile and chalcophile character, when most moderately volatile elements are partially lithophile. Thus, Sn has the potential to track metal-sulphide segregation and devolatilisation events. Here we present new Sn concentration and isotopic data for the LL4 chondrite Bjurböle. As a LL4, we know it has experienced thermal processing. Sn isotope analysis has been performed for bulk chondrite samples along with analyses for separated chondrules and matrix. This allows for testing of the hypothesis of complementarity and degree of heterogeneity and thermal processing of the chondrules themselves.

Differences in $\mathrm{Sn}$ concentration and $\delta^{122 / 118} \mathrm{Sn}$ are observed between chondrules and matrix. Ranges in Sn concentration for individual chondrules are 0.86 and $8.8 \mu \mathrm{g} \mathrm{g}^{-1}$; and $-0.58 \%$ and $2.63 \%$ for $\delta^{122 / 118} \mathrm{Sn}$. Matrix Sn concentration ranges from 12.28 to $23.42 \mu \mathrm{g} \mathrm{g} \mathrm{g}^{-1}$ and $1.59 \%$ to $2.04 \%$ o $\delta^{122 / 118} \mathrm{Sn}$. The bulk chondrite value $\left(3.55 \mu \mathrm{g} \mathrm{g}^{-1}, \delta^{122 / 118} \mathrm{Sn}=0.10 \pm 0.03 \%\right.$ ) occupies an intermediate position between the bulk chondrule value and the matrix values. Comparison of the $\mathrm{Sn}$ values with those from a study on tektites indicates the fractionation of $\mathrm{Sn}$ isotopes is not entirely controlled by volatilisation. This relationship of Sn elemental and isotopic compositions between chondrules and matrix suggests Sn complementarity between these phases. Complementarity in chondrites would require that the chondrules and matrix formed in the same spatial region of the early Solar System, placing significant constraints on chondrule formation models. Additionally, Bjurböle chondrules range from glassy to highly porphyritic in texture, and the individual chondrule analyses for $\mathrm{Sn}$ show a range of values, with one having $\delta^{122 / 118} \mathrm{Sn} 2.62 \pm 0.05 \%$ and is isotopically heavier than that of both other individual chondrules and matrix. This variation shows that $\mathrm{Sn}$ isotopic fractionation is heterogeneous. Given this observed heterogeneity in the chondrules, chondrule formation models must also satisfactorily explain the textural variability in chondrules. Loss of Sn and fractionation of its isotopes may be tied to the same process that produced the varying textures of chondrules within Bjurböle. 